\title{
Active Noise Control proposal design enhanced because of using sensors/actuators based on nanostructures
}

\author{
Jesus A. Calderón ${ }^{1,2, *}$, Julio C. Tafur ${ }^{2}$, Eliseo B. Barriga ${ }^{2}$, Roland Mas $^{2}$, Luis Chirinos ${ }^{2}$, Enrique Barrantes ${ }^{2}$, \\ Jorge Alencastre ${ }^{2}$, Oscar Melgarejo ${ }^{2}$, John H. Lozano ${ }^{2}$, Bjorn Heinrich ${ }^{1,2}$, Enrique Aguilar ${ }^{2}$, Juan C. Lengua ${ }^{2}$
}

${ }^{1}$ TU Ilmenau University of Technology, Institute of Physics and IMN MacroNano, Germany

${ }^{2}$ Pontificia Universidad Católica del Perú Energy Laboratory and Mechatronic Departament, Perú

\begin{abstract}
Big machines and motors, that under operating work produces high decibels of noise in wide range of work, these values are near $90 \mathrm{~dB}$ that is not healthy for humans. Normally, it is used passive mechanisms to attenuate noise such as big headphones. However that solution is not enough when noise has changes in frequency domain. Therefore, it is proposed in this work a solution by noise cancellation with Active Mechanisms; nevertheless, we designed a hybrid algorithm improved through predictive/adaptive concurrent algorithms strategies, with Active Noise Cancellation (ANC). By other side, based on nanostructures, it has been analyzed the effect in robustness and wide range of work by frequency domain in order to enhance noise cancellation.
\end{abstract}

\section{Introduction}

Plenty ANC strategies are applied nowadays in order to attenuate noise from wide frequency domain instead of passive mechanisms, which are mostly expensive due to quality of material to absorb noise on the place to look for noise cancellation, furthermore geometry characteristics of the place change owing to add that noise absorber material. Therefore, many techniques and researches are applied actually, however, many times, it provokes high computing cost, specially to propose adaptive algorithms to get better predictions in order to achieve an efficient noise cancellation,that can be solved with different software strategies, but as it implies computing cost, it means sampling time tends to be bigger than response time of the system where to get noise cancellation. By other hand, it is proposed in this work the necessity to use nanostructures which could be the base to elaborate sensors and actuators, because of achieving wide range of work in frequency domain to register information from sensors (based on nanostructures) and to send noise cancellation signal with low possibility to be disturbed through actuators (based on nanostructures).

In this work was evaluated high decibels noise from a Diesel Motor through Least Mean Square (LMS) adaptive coefficients, it because to get good performance when environment conditions (air temperature and air pressure) are not stable, furthermore, it was analyzed the residual noise around the scope of work. In figure 1, it is depicted a Diesel Motor (Energy Laboratory, PUCP) from which was attenuated noise by the algorithms and strategies designed in this work.

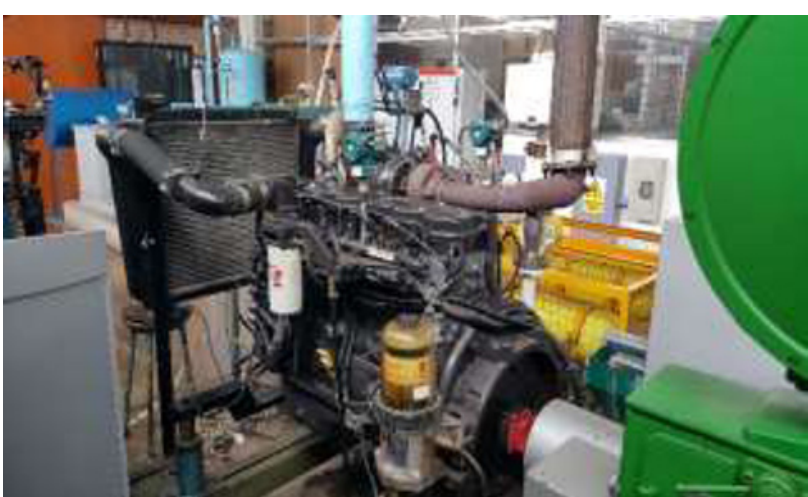

Fig 1. Main Disel Motor for experimental tests, Energy Laboratory of Pontificia Universidad Católica del Perú.

\section{Active Noise Control ANC}

There are many mechanisms to get ANC, such as feedback, feedforward and hybrid (between last both) methodologies, that can be obtained (also improved) by positioning in strategic positions the sensors/actuators: "microphones and loudspeakers".

Usually, the primary signal should be attenuated by a secondary signal which should be slightly the same primary signal but inverse, in order to generate the same pressure of sound [2], [10], [12].

It is possible to design algorithms with the objective to achieve a better error signal as a dependency of the weights of the system. In following equation is represented a general mechanical wave equation, according to represent theoretically sound propagation [9]. 
Where, $q(x, t)$ represents the sound source, $P(x, t)$ represent pressure field, $\mathrm{X}$ is the space variable, $\mathrm{c}$ represents the speed of sound, $t$ is the time variable, and r2 is the Laplacian.

$$
\frac{1}{c^{2}} \frac{\delta^{2}[P(x, t)]}{\delta t^{2}}-\nabla^{2}[P(x, t)]=q(x, t)
$$

Outside the space domain, if it is assumed that the sound source $\mathrm{q}(\mathrm{x}, \mathrm{t})$ is null, it could be achieved while an external signal is opposite to $\mathrm{q}(\mathrm{x}, \mathrm{t})$, ideally in amplitude and frequency. Nevertheless, due to many factors such as computing cost, robustness and short time response of sensors/actuators (microphones/loudspeakers), the objective is probably difficult to achieve. Otherwise, it is consequently necessary to analyse strategies with estimation "E ", error analysis "e" (as dependence of desired signal " $\mathrm{d}$ " and measured signal " $\mathrm{m}$ "), as it was made in this work through costing function " $\mathrm{J}$ ", in discrete time " $n$ ".

$$
J(n)=(E(d(n)-m(n)))^{2}
$$

\subsection{Adaptive FXLMS algorithm}

For analysis made in this work, an adaptive FXLMS algorithm was evaluated because many successful results were obtained by this kind of algorithm, in which it is frequently to estimate weights "W" to be adapted every instant " $n$ " in the input signal " $X$ " in order to get an optimal error. Motor noise are usually a mixing of harmonic functions, no matter whether it is an "Electrical Motor or Diesel Motor" and of course changes produced in their amplitudes and frequencies are due to some specific characteristics through their physical parameters as well as the energy sources involved (such as combustion or some kind of electrical/mechanical energy transformation). It means that physical parameters can change as a consequence of external physical conditions, therefore, changes could produce an unsuitable adjustment during control. For this reason, it is possible to solve it by control strategies (with high computing time disadvantages) or through analysis using systems identification algorithm, such as classic FXLMS (owing to good recurrence in the main algorithm). However, as the costing function can be adapted by its "weights", the solution can be enhanced as it was probed in this work, achieving a general algorithm for motors in operating range from $40 \mathrm{~dB}$ to $90 \mathrm{~dB}$ and motor speed from 500 RPM to 3000 RPM.

In figure 2, it is shown a representation of the costing function curve that has changes dependences which means that through derivative of "J" with respect to the weight " $\mathrm{w}$ " is possible to find a minimal value around " $\mathrm{J}$ " [5], [6].

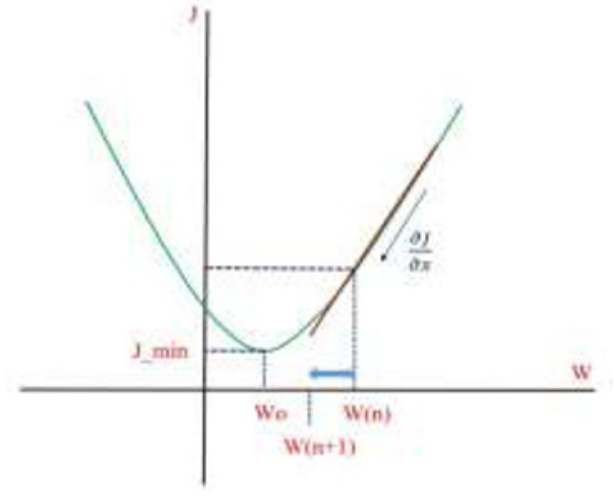

Fig. 1. Costing function representation for ANC analysis an adaptive effect of weights

It means that in the classic FXLMS algorithm (as it is represented in following equation) for the secondary path "S" (that is looking the reflection effect after sending the antinoise signal) every adaptive coefficient " $w$ " can be adjusted in a better way in order to get the optimal error. Notwithstanding, it is good proposal to start predictive criterion, while it could be necessary as function of costing function.

$$
e(n)=d(n)-S(n) *\left[w^{T}(n) X(n)\right]
$$

\subsection{Hybrid Controller}

With the main objective to get the noise cancellation, the main control is designed through a hybrid configuration between feedback/ feedforward distribution [1], as the one depicted in figure 3 where " $x$ " is the input noise signal and " $d$ " is the desired signal obtained after to cross the physical space "P" (which is known as primary path). The block "LMS" is the Least Mean Square (function in algorithm connotation) in which the adaptive weights are contained to get the adaptive noise cancellation. This is achieved through the right matrices in adaptive coefficients "A and " $C$ " which depends on the feedback and feedforward configuration controlling the error through the secondary path effect " $\mathrm{S}$ " for every instant "n".

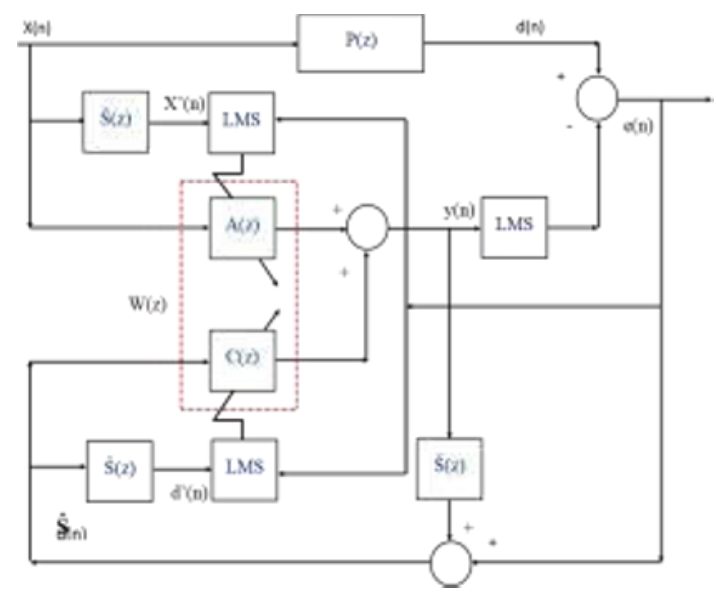

Fig 2. Hybrid block diagram scheme [3] 


\section{Simulations and Analysis}

With all theoretical analysis described above, the algorithm was designed according to get noise cancellation for harmonic signals with changes in frequency domain as it is expected in motors noise evaluations. However, a simple classic search is always made by testing the opposite signal to the main noise signal. For this reason, in figure 4 is shown part of motor noise signal (Diesel motor - Energy Laboratory of PUCP) and antinoise signal that was achieved after sending the opposite signal to the space domain where the motor was located. The average noise was $90 \mathrm{~dB}$ at $1000 \mathrm{RPM}$ (in figure 4 it is shown its equivalent in Voltage as it was obtained by microphones). The antinoise signal accomplished a cancellation of $10 \mathrm{~dB}$ approximately; it was because the motor did not increase speed. Therefore, computing time was enough in order to send the simple opposite signal. Nevertheless, when the motor speed changed, the error increased. For this reason, it is suggested to design an adaptive hybrid algorithm based in FXLM.

On the other hand, LMS is evaluated to adapt the main control algorithm. For this reason, expected results are shown in figure 5 . A reduction of $50 \mathrm{~dB}$ (voltage equivalence) is achieved.

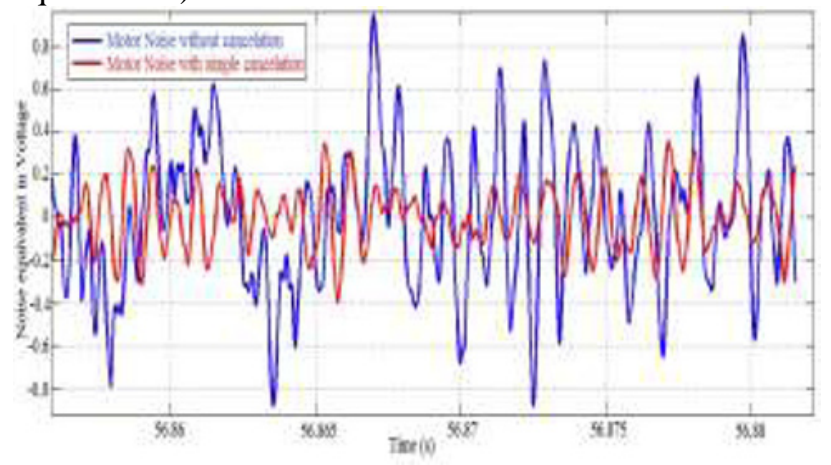

Fig. 3. Motor noise signal and simple noise cancellation based on ANC

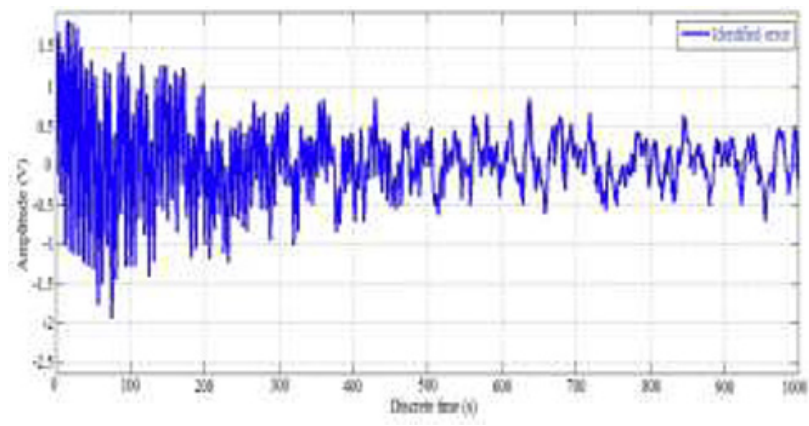

Fig. 4. LMS results

Evaluation was made positioning the antinoise source only around this physical space and the noise was attenuated. However, that is not usual while workers are around the room where the motor is operated. For that reason, all the physical space was analysed in order to get a better spatial noise cancellation, so that it was generalized a source " $\mathrm{M}$ " (as it is described in figure 6) that produce noise for every position " $r$ ". Also there are represented " $\mathrm{m}$ " antinoise sources " $\mathrm{NC}$ " which try to get desired noise cancellation at every instant " $n$ ".

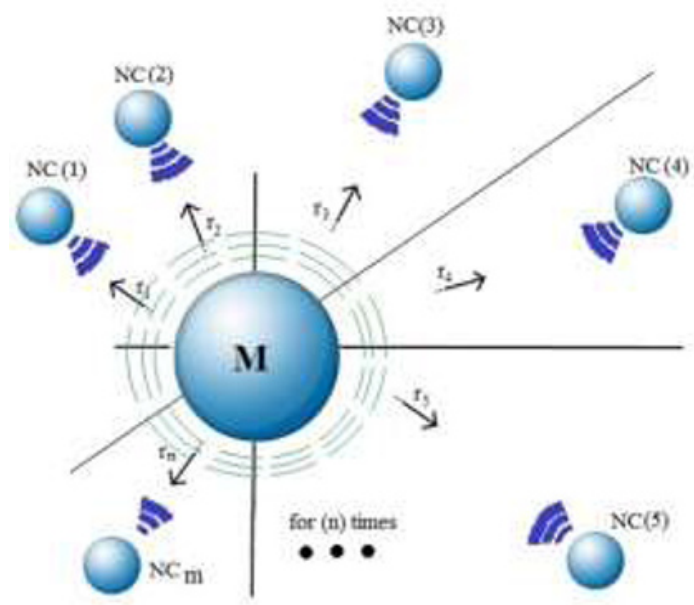

Fig. 6. 3D mathematical representation for ANC that is generalized for " $n$ " antinoise sources.

Applying the adaptive algorithm:

$$
e(n)_{y}=d(n)_{y}-S(n)_{y}
$$

For every

$$
\begin{aligned}
S(n)_{y}= & S(n)_{i} *\left[w^{T}(n)_{i} X(n)_{i}\right] \\
e(n)_{y}= & \left(\begin{array}{c}
e(n) \\
e(n) \\
\vdots \\
e(n)
\end{array}\right)
\end{aligned}
$$

Then, after identifying the motor noise by LMS, it was possible to evaluate the $3 \mathrm{D}$ noise cancellation simulating 3 antinoise sources for the primary source. In average the expected noise reduction was between $50 \mathrm{~dB}$ to $52 \mathrm{~dB}$ as it is depicted in figure 7 . However, it is suggested to use a controller which could be faster enough than the motor noise changes (as real time controllers).

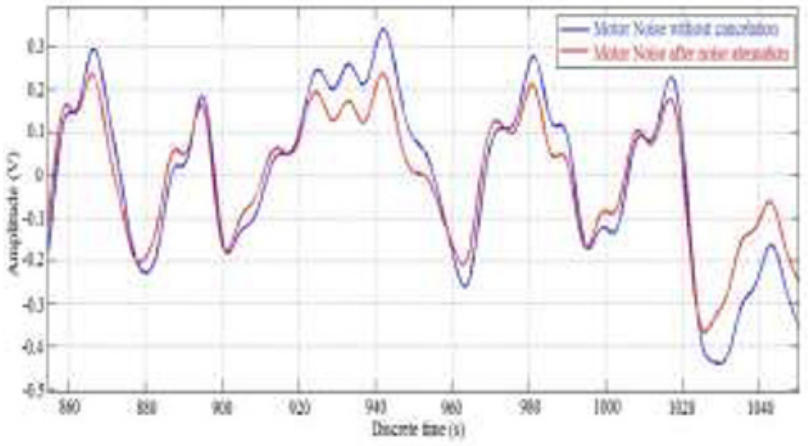

Fig. 5. Motor noise signal and noise cancellation based on LMS adaptive algorithm

\section{Enhancement Proposal}

From last results, through simulations and experiments, response time is an important factor to achieve noise cancellation. Moreover, to get enough time to solve task 
by the main controller such as adaptive cancellation logic. For this reason, it is proposed in this work to replace traditional actuators (loudspeakers) by sound emission based on nanostructures due to the fact that sensors and actuators based in high order nanostructures arrays provide faster response time and robustness. Figure 8 shows motor noise attenuated using ANC strategies and sensors/actuators based on nanostructures.

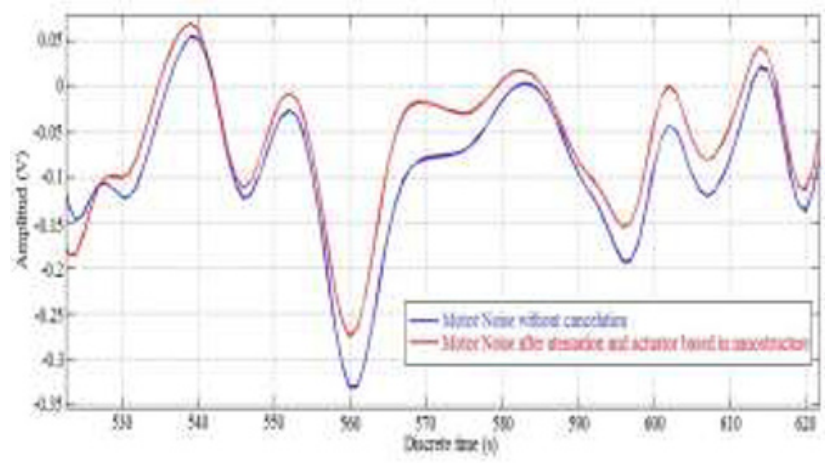

Fig. 6. Motor noise signal and cancellation considering nanostructure effect.

\section{Conclusions}

In this work, a hybrid-adaptive algorithm was evaluated in order to get noise cancellation that was produced by a diesel motor of $10 \mathrm{HP}$. Noise cancellation was achieved reducing noise from $80 \mathrm{~dB}$ to $37 \mathrm{~dB}$.

It was analysed a $3 \mathrm{D}$ propagation $\mathrm{ANC}$ due to geometric dependence between sensors/actuators (microphones/loudspeakers) and noise source.

Through simulations, it was obtained that sound transmitter based on nanostructures produces faster responses to get optimal noise cancellation that also is a good support to achieve predictions.

The author wants to thank Mr. César José Leclere Mota, Bruno Sebastián Miranda Quispe, José Miguel Pérez Flores and Arturo Emilio Berastain Hurtado because of their support in experimental tests.

\section{References}

1. J. A. Calderón, «Analysis and Implementation of Active Noise Control Strategies using Piezo and EAP Actuators,» PUCP \& TU Ilmenau, 2015.

2. K. C. Zangi, «Optimal Feedback Control Formulation of the Active Noise Cancellation Problem: Pointwise and Distributed,» Massachusetts Institute of Technology, Diss, 1994.

3. J. A. Calderón, «Analysis of Online Active Noise Control Strategies by Hyrid Algorithm,» PUCP, 2016.

4. Z. Dabrowski, «Methodology of Selecting The Reference Source for an Active Noise Control System in a Car,» International Journal of Occupational safety and Ergonomics, 2013.

5. S. M. Kuo y D. R. Morgan, «Active Noise Control: A Tutorial Review,» Proceedings of the IEEE, 1999.
6. D. R. Morgan, «History, Applications and Subsequent Development of the FXLMS Algorithm,» IEEE Signal Processing Magazine, 2013.

7. S. Simranjit, «Implementation of Active Noise Cancellation in a Duct,» 2013.

8. M. Larsson, «Active Control of Noise in Ventilation Systems Analysis and Experiments,» Blekinge Institute of Technology, 2011.

9. A. Oey, «FXLMS ANC, Matlab Central,» 2311 2010. [En línea]. Available: https://www.mathworks.com/matlabcentral/fileexcha nge/27277-active-noise-control-system-using-fxlmsalgorithm.

10. N. Ravikanth, «Design and Development of Noise Cancellation System for Android Mobile Phones,» SAS TECH, 2012.

11. R. Bartholomae, «Active Noise CancellationPerformance in Hearing Protector Under Ideal and Degraded Conditions,» United State Department of the Interior, Bureau of Mines, 1994.

12. «Audio Techinica,» 2015. [En línea]. Available: http://eu.audio-technica.com/en/. 